\title{
Transcranial static magnetic field stimulation of M1 reduces corticospinal excitability without distorting sensorimotor integration in humans
}

\author{
Pablo Arias ${ }^{1}$, Lucía Adán-Arcay ${ }^{1}$, Beatriz Puerta-Catoira ${ }^{1}$, Antonio Madrid ${ }^{1}$, \\ Javier Cudeiro ${ }^{1,2}$ \\ ${ }^{1}$ Universidade da Coruña, NEUROcom, Dept. Medicine-INEF, INIBIC, Spain \\ ${ }^{2}$ Centro de Estimulación Cerebral de Galicia, A Coruña, Spain
}

Keywords

Motor cortex; Excitability; Magnetic fields; Sensorimotor integration

Dear Editor:

Transcranial static magnetic stimulation (tSMS) over the human motor cortex using powerful neodymium magnets induces a reduction in corticospinal excitability. It seems to act through intracortical inhibitory (ICI) mechanisms, which are $\mathrm{GABA}_{\mathrm{A}}$-dependent [1] ; [2]. Similar effects have been documented in somatosensory cortex [3]. Given that tSMS modulates motor and sensory physiology [3], we wondered whether static magnetic fields could also affect sensorimotor integration. Here, we evaluated the effects of tSMS over M1 on short/long afferent-inhibition (SAI/LAI). SAI/LAI occur at cortical level when coupling (with the proper timing) an electrical pulse on a peripheral nerve (PNS) with a transcranial magnetic stimulation (TMS) pulse on M1 [4]. SAI is known to interact with ICI [5], therefore we hypothesized that tSMS-M1 would reduce corticospinal excitability with after-effects on SAI.

Seventeen un-medicated, healthy people participated (20-41yrs) (Ethic-Committee approval: CE-17/2015). In each session we determined (individually) the inter-stimulus-interval (ISI) which was used to evaluate SAI by recording 70 TMS motor-evoked potentials (MEP) in the relaxed first dorsal interosseous (FDI) muscle, at $0.2 \mathrm{~Hz}$. Ten MEP were unconditioned at an intensity high enough to obtain response amplitudes $\approx 1 \mathrm{mV}$, and 60 conditioned with PNS on the median nerve at the wrist, at the following intervals: 20-21-22-23-24-25 ms (Fig. 1a). Then, 36 pre-MEP were recorded. Of these, twelve were unconditioned (TEST) with a fixed intensity producing amplitudes $\approx 1 \mathrm{mV}$, and the other 24 conditioned by PNS (12 to evaluate SAI at the ISI determined above, and 12 to evaluate LAI with ISI $=200 \mathrm{~ms}$ [6]). MEP were acquired in 12 sequences (TEST-SAI-LAI). Next, tSMS was applied on the M1-FDI hot-spot as detailed previously [1], for 20min. One session was real tSMS (magnetic field of $0.5 \mathrm{~T}$ ), the other sham, with the order counterbalanced across subjects. Then, Post-MEP were recorded in two blocks: i) Post-fixed intensity (TMS intensity was the same as pre) and ii) post-matched amplitude (TMS intensity was adjusted to obtain TEST-amplitudes $\approx$ pre-TEST); again the order was counterbalanced across subjects. Finally, we followed with the post 2 evaluation, starting $15 \mathrm{~min}$ after the end of tSMS. The procedure was the same as in post. 
a

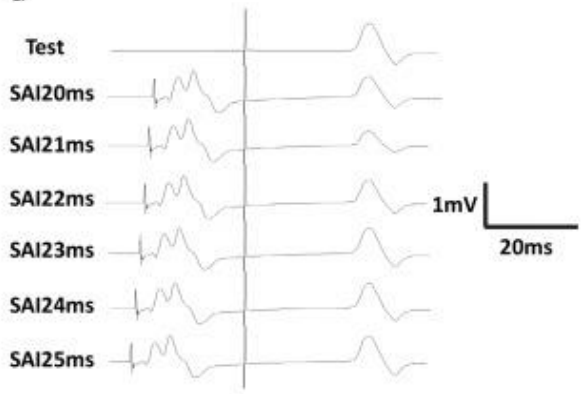

C

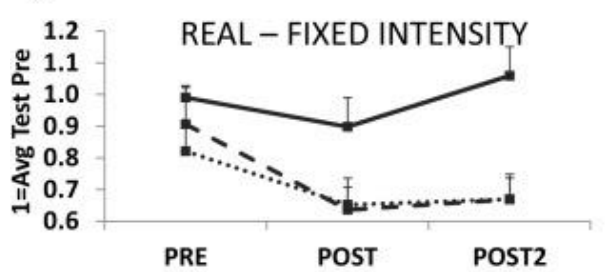

e

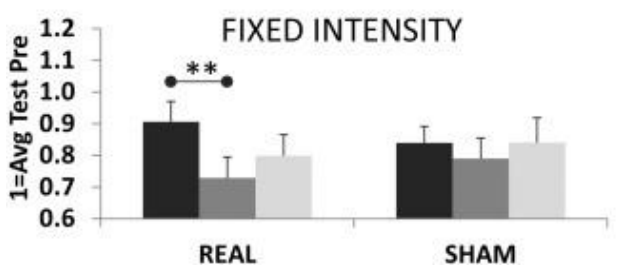

b

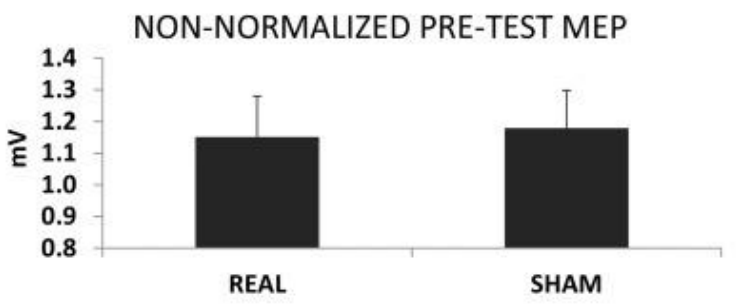

d

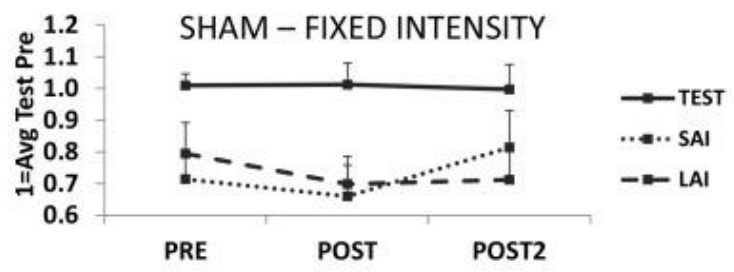

f

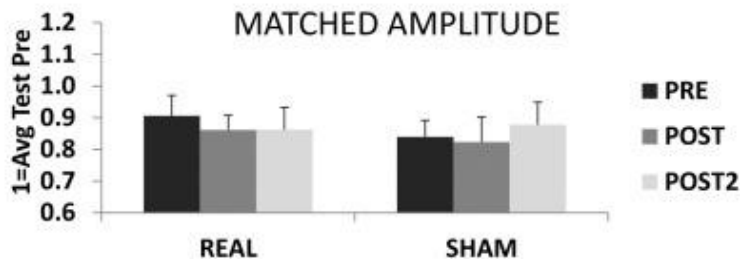

Fig. 1. a.) SAI inter-stimulus-interval (ISI) determination in one subject (an ISI of $21 \mathrm{~ms}$ was chosen because it generated the greatest inhibition). b.) Non-normalized TEST amplitudes obtained during real and sham sessions did not differ at pre (Student t-test). The average of pre values in real and sham sessions was $1.16 \mathrm{mV}$ (SE 0.12); this value is used as the unitary value in the ordinate of the graphs shown in the rest of the figure. c,d.) TEST, SAI and LAI changes over testing times (for fixed-intensity blocks) were different for real and sham (ANOVA $\mathrm{p}=0.035_{\text {STIMxCONDITIONxTIME). Subsequent }}$ ANOVA for each stimulation mode (real and sham) showed a reduction of MEP over time for real-tSMS (ANOVA $\mathrm{p}=0.016_{\mathrm{TIME}}$ ) observed for TEST, SAI and LAI (ANOVA $\mathrm{p}>0.05_{\mathrm{TIMExCONDITION}}$ ); at post MEP were reduced $\approx 20 \%$ (post$h o c ; \mathrm{p}=0.005, \mathrm{p}=0.015_{\text {Bonferroni }}$ ). For sham-tSMS MEP did not change significantly over time. e) Time effects for $\mathrm{c}$.) and d.) with all Conditions pooled. f) MEP responses pooling Conditions for matched-amplitude blocks on a time basis. In all analyses shown in figures $\mathrm{c}-\mathrm{f}$, the amplitude of the TEST was always larger than SAI and LAI (ANOVA

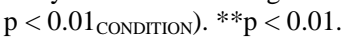

For TMS application and MEP recording we followed standard methods described elsewhere [1]. PNS was applied with a Digitimer-DS7A $(500 \mu \mathrm{sec}$ pulse-duration; cathode proximal). MEP amplitudes were normalized (intra-subject normalization). For this, for each subject we calculated the average of the values of the TEST at pre across the real and sham sessions. This value was used as denominator for all values of the given subject at all testing time-points, either for the TEST, SAI and LAI. Graphs show means and standard error of the mean (SE). Results were considered significant if $\mathrm{p}<0.05$.

The essential message from our results is straightforward: tSMS reduces corticospinal excitability but has no effect on SAI or LAI. Fig. 1b shows similar non-normalized TEST amplitudes for real and sham sessions before magnet application (similar results were obtained for SAI and LAI). For fixed-intensity blocks, real-tSMS reduced the normalized amplitudes of the TEST, SAI and LAI at post (by $\approx 20 \%$ ), but all recovered at post 2 ; sham-tSMS produced no effects ( Fig. 1c-e). To check whether SAI and LAI responses to tSMS were influenced by the size of the TEST, responses to tSMS were acquired while keeping the amplitude of the TEST constant at pre, post and post 2 (matched-amplitude blocks). In this case the amplitude of TEST, SAI and LAI did not change significantly over time ( Fig. 1f, TIME effect; all conditions pooled). In fixedintensity and matched-amplitude blocks TEST were larger than SAI and LAI at all testing times. 
These results corroborate the finding that $20 \mathrm{~min}$ of tSMS reversibly reduces corticospinal excitability [1]. tSMS affected SAI and LAI similarly, and both followed the same pattern of inhibition shown affecting TEST. However, the evaluation of SAI and LAI while maintaining the same amplitude of the TEST along testing-times (matched-amplitude blocks) reveals that SAI/LAI were not modified by tSMS. Therefore, tSMS reduces corticospinal excitability without affecting SAI and LAI.

The mechanisms underlying SAI are mainly cortical and are related to the cholinergic and GABAergic systems [5] ; [7]. The fact that in our hands tSMS seems not to directly affect SAI circuits but has been shown to modulate ICI [2] leads us to conclude that SAI and ICI might be representing the operational mechanism of distinct subtypes of GABAergic inhibitory interneurons [8], which in one case is affected by tSMS, but not in the other. We cannot discount an effect of tSMS on intrinsic excitability of the corticospinal motoneurons; in the case of a putative inhibition of M1 pyramidal cell bodies the response to TMS would wane independently of the input to the cells from cortico-cortical connections, something compatible with our results. The mechanisms responsible for LAI are less understood but it has been proposed that LAI implicates interneurons acting via $\mathrm{GABA}_{\mathrm{B}}$ receptors. Nevertheless, whatever the exact mechanism, it seems to be unaffected by tSMS on M1.

In conclusion, tSMS-M1 reduces corticospinal excitability without affecting SAI and LAI. Further research should explore the effect of tSMS on some other expressions of sensorimotor integration.

\section{Acknowledgment}

We thank Dr. Ken Grieve for revising the manuscript. Grants: Xunta de Galicia. Grupos Consolidados 2014.

\section{References}

[1]. A. Oliviero, L. Mordillo-Mateos, P. Arias, I. Panyavin, G. Foffani, J. Aguilar. Transcranial static magnetic field stimulation of the human motor cortex. J Physiol Lond, 589 (20) (2011), pp. 4949-4958.

[2]. I. Nojima, S. Koganemaru, H. Fukuyama, T. Mima. Static magnetic field can transiently alter the human intracortical inhibitory system. Clin Neurophysiol, 126 (12) (2015), pp. 2314-2319.

[3]. H. Kirimoto, H. Tamaki, T. Matsumoto, K. Sugawara, M. Suzuki, M. Oyama, et al. Effect of transcranial static magnetic field stimulation over the sensorimotor cortex on somatosensory evoked potentials in humans. Brain Stimul, 7 (6) (2014), pp. 836-840.

[4]. H. Tokimura, V. Di Lazzaro, Y. Tokimura, A. Oliviero, P. Profice, A. Insola, et al. Short latency inhibition of human hand motor cortex by somatosensory input from the hand. J Physiol, 2 (523 Pt) (2000), pp. 503-513.

[5]. H. Alle, T. Heidegger, L. Krivanekova, U. Ziemann. Interactions between short-interval intracortical inhibition and short-latency afferent inhibition in human motor cortex. J Physiol, 587 (Pt 21) (2009), pp. 5163-5176.

[6]. R. Chen, B. Corwell, M. Hallett. Modulation of motor cortex excitability by median nerve and digit stimulation. Exp Brain Res, 129 (1) (1999), pp. 77-86.

[7]. V. Di Lazzaro, A. Oliviero, P. Profice, M.A. Pennisi, S. Di Giovanni, G. Zito, et al. Muscarinic receptor blockade has differential effects on the excitability of intracortical circuits in the human motor cortex. Exp Brain Res, 135 (4) (2000), pp. 455-461.

[8]. V. Di Lazzaro, F. Pilato, M. Dileone, P. Profice, F. Ranieri, V. Ricci, et al. Segregating two inhibitory circuits in human motor cortex at the level of GABAA receptor subtypes: a TMS study. Clin Neurophysiol, 118 (10) (2007), pp. 2207-2214. 